\title{
ESTIMATES FOR SOLUTIONS OF \\ ELLIPTIC EQUATIONS IN A LIMIT CASE
}

\author{
Ester GiarRusso and Guido Trombetti
}

Let $u$ be a weak solution of the homogeneous Dirichlet problem for a second order elliptic equation of divergence form, in a bounded open subset of $\mathbb{R}^{n}$. We prove, that if the right hand side of the equation is an element of $H^{-1, n}(\Omega)$, then $u$ belongs to the orlicz space $L_{\Phi}$ where $\Phi(t)=\exp \left(|t|^{n /(n-1)}\right)-1$. We employ the properties of the Schwartz symmetrization thus obtaining the "best" constant of the estimate.

\section{Introduction}

Denote by $\Omega$ a bounded open subset of $\mathbb{R}^{n}(n \geq 2)$ and consider the Dirichlet problem

$$
\begin{cases}-\left(a_{i j}(x) u_{x_{i}}{ }^{\prime} x_{j}\right. & =-\left(f_{j}^{\prime} x_{j} \text { in } \Omega\right. \\ u=0 & \text { on } \partial \Omega .\end{cases}
$$

Received 27 November 1986. Supported in part by M.P.I. (408).

Copyright Clearance Centre, Inc. Serial-fee code: 0004-9729/87 $\$ A 2.00+0.00$. 425 
Here the coefficients $a_{i j}$ are bounded and measurable, the $f_{j}$ are in $L^{p}(\Omega), p \geq 2$, and

$$
a_{i j}(x) \xi_{i j} \xi_{j} \geq|\xi|^{2} \quad x \in \Omega, \quad \xi \in \mathbb{R}^{n} .
$$

Here and below the summation convention is employed. Put

$$
f=\left(\sum_{i=1}^{n}\left|f_{i}\right|^{2}\right)^{1 / 2}
$$

It is very well known ([16]) that

$$
\|u\|_{p^{*}} \leq K(p, n)\|f\|_{p} \quad p^{*}=\frac{n p}{n-p} \text {, if } 2 \leq p<n \text {, }
$$

and that

(1.3) . $\|u\|_{\infty} \leq K(p, n)|\Omega|^{\frac{1}{n}-\frac{1}{p}}\|f\|_{p}$ if $p>n$.

Actually, this is the Sobolev imbedding theorem if we replace $f$ by $|D u|$.

For the limit case $p=n$, Stampacchia [17] showed that the solution $u$ of (1.1) is in the orlicz space $L_{\Phi}$ defined by the function $\Phi(t)=e^{|t|}-1$. (See [1] and [11] for the exact definition and the properties of orlicz spaces). On the other hand, Trudinger proved later that $W^{1, n}(\Omega)$ is continuously imbedded in the orlicz space $L_{\Phi}$ defined by $\Phi(t)=\exp \left(|t|^{n /(n-1)}\right)-1,([20]$, Theorem 2, p.478). Hence one can expect that Stampacchia's result can be sharpened and that the solution $u$ of (1.1) is in the same Orlicz space as that found by Trudinger (that is with $\left.\Phi(t)=\exp \left(|t|^{n /(n-1)}\right)-1\right)$.

The aim of this paper is to prove the following estimate

$$
\|u\|_{\Phi} \leq c(n,|\Omega|)\|f\|_{n}
$$

where

$$
\|u\|_{\Phi}=\inf \left\{k>0: \int_{\Omega} \Phi\left(\frac{u}{k}\right) d x \leq 1\right\}
$$

and

$$
\Phi(t)=\exp \left(|t|^{n /(n-1)}\right)-1
$$


We actually get a slightly better result. We show that

$$
[u]_{1-1 / n} \leq\left(n c_{n}\right)^{-1 / n}\|f\|_{n},
$$

where

$C_{n}=$ the measure of the unit ball of $\mathbb{R}^{n}$. Put

$\Omega^{\#}=$ the ball of $\mathbb{R}^{n}$ centred at the origin and with the same measure as $\Omega$,

$r=\left(|\Omega| / C_{n}\right)^{1 / n}=$ the radius of $\Omega^{\#}$,

$u^{\#}(x)=$ the spherically decreasing symmetric rearrangement of $u$ (see

Section 2).

Moreover, the value of the constant on the right-hand side of $(1.6)$ is the best possible. The quantity

$$
[u]_{1-1 / n}=\sup _{\Omega_{0}^{\#}} u^{\#}(x) /\left(\log \frac{r}{|x|}\right)^{1-1 / n}
$$

has been introduced in [2], where a "sharp" version of Trudinger's imbedding theorem is given. A paper also related to this topic is [13].

In [2] it is proved that $\forall u \in \|^{1, n}(\Omega)$

$$
[u]_{1-1 / n} \leq\left(n c_{n}\right)^{-1 / n}\|D u\|_{n}
$$

and the constant is the best one. The connection between (1.5) and (1.7) is given by the following inequality ([2])

$$
\|u\|_{\Phi} \leq\left(\frac{1+|\Omega|}{n}\right)^{1-1 / n}[u]_{1-1 / n}
$$

We finally point out that more generally our result applies to elliptic operators of degenerate type like those in [14] and in [21]. For this kind of operator the estimate (1.4) has already been obtained by Trudinger for the case $n=2,([21$, Theorem 4.1]).

2. Hypotheses and preliminaries

Throughout this paper we assume

$$
\begin{aligned}
& a_{i j}(x) \xi_{i} \xi_{j} \geq m(x)|\xi|^{2} \text { a.e. in } \Omega, \xi \in \mathbb{R}^{n} \\
& m(x) \geq 0 \text { in } \bar{\Omega} \\
& m(x) \in L^{s}(\Omega), m^{-1}(x) \in L^{q}(\Omega), \frac{1}{s}+\frac{1}{q} \leq \frac{2}{n} \\
& a_{i j} m^{-1} \in L^{\infty}, f_{j} m^{-1 / p} \in L^{p}(\Omega), p \geq 2 .
\end{aligned}
$$


We also use the following notation.

Given a measurable real valued function $f$ in $\Omega$, the distribution function of $f$ is

$$
\mu(t)=\operatorname{meas}\{x \in \Omega:|f|>t\} ;
$$

the decreasing rearrangement of $f$ is

$$
f^{*}(s)=\inf \{t \geq 0 ; \mu(t)<s\} ;
$$

and the spherically symetric rearrangement of $f$ is

$$
f^{\#}(x)=f^{*}\left(C_{n}|x|^{n}\right)
$$

Finally, for $f \in L^{p}(\Omega)$ we denote by

$$
\begin{gathered}
K(f)=\left\{F \in L^{P}(0,|\Omega|) \text { for which } Z\left\{f_{k}\right\} \subset L^{p}(0,|\Omega|):\right. \\
\left.f_{k}^{*}=f^{*} \text { and } f_{k} \rightarrow F \text { in } L^{p}(0,|\Omega|)\right\} .
\end{gathered}
$$

Now we state a lemma of weak approximation, which is one of the main tools for the proof of Theorem 3.1.

Consider the distribution function $\mu(t)$ of the solution $u(x)$ of (1.1) and for any $s \in[0,|\Omega|]$ a subset $D(s)$ of $\Omega$ such that

$$
\begin{aligned}
& \text { meas } D(s)=s \\
& s_{1}<s_{2} \Rightarrow D\left(s_{1}\right) \subset D\left(s_{2}\right) \\
& D(s)=\{x \in \Omega:|u|>t\} \quad \text { if } s=\mu(t) .
\end{aligned}
$$

Hence, given a function $f \in L^{p}(\Omega)$, there exists a function $F(t)$ such that

$$
\int_{D(8)} f(x) d x=\int_{0}^{s} F(t) d t .
$$

LEMMA 2.1. The function $F(t)$ given by (2.1) belongs to $K(f)$. See the proof in [4]. See also [12] and [15] for a similar topic.

3. The estimate for the limit case

To get our result we use a symmetrization technique ([4], [5]. [10], [18]). First we prove the following comparison lemma.

LEMMA 3.1. Let (i) - (ii) be satisfied and let $u$ be the solution of (1.1). Then

$$
u^{\#}(x) \leq v(x)
$$


where $v(x)$ is a solution of the problem

$$
\left\{\begin{array}{l}
-\left(\underline{m}\left(C_{n}|x|^{n}\right) v_{x_{i}}{ }^{x_{i}}=\left(F\left(C_{n}|x|^{n}\right)^{1 / 2}\left(C_{n}|x|^{n}\right) x_{i} /|x|\right)_{x_{i}} \text { in } \Omega^{*}\right. \\
v=0 \text { on } \partial \Omega^{*},
\end{array}\right.
$$

$F^{2}(s) \in K\left(f^{2} / m\right)$ and $\underline{m}^{-1}(s) \in K\left(m^{-1}\right)$.

Proof. Since $u \in H_{0}^{1}(m)$ (where $H_{0}^{1}(m)$ is (see [14]) the completion of $C_{0}^{\infty}(\Omega)$ with respect to the norm

$$
\left.\|u\|_{H_{0}^{1}(m)}=\left(\int_{\Omega} m(x)|D u|^{2} d x\right)^{1 / 2}\right)
$$

is a weak solution of (1.1), then

$$
\int_{\Omega} a_{i j} u_{x_{i}} \psi_{x_{j}} d x=\int_{\Omega} f_{j} \psi_{x_{j}} d x \quad \forall \psi \in H_{o}^{1}(m) .
$$

Now choose in the above

$$
\psi(x)=\left\{\begin{array}{ccc}
(|u|-t) \operatorname{sgn} u & \text { if } & |u|>t \\
0 & \text { if } & |u| \leq t
\end{array}\right.
$$

to obtain

$$
\int_{|u|>t} a_{i j} u x_{i} u_{x_{j}}=\int_{|u|>t} f_{j} u_{x_{j}} d x, t>0 .
$$

Recall (i) and perform some standard calculations ([4], [18]) to get

$$
-\frac{d}{d t} \int_{|u|>t} m(x)|D u|^{2} d x \leq-\frac{d}{d t} \int_{|u|>t} \frac{f^{2}(x)}{m(x)} d x .
$$

Now, with the same technique as that used in [4], [18], by the De Giorgi isoperimetric inequality ([6]) and a Fleming-Rishel formula ([7]), we have

$$
\left.n C_{n}^{1 / n_{\mu(t)}}\right)^{1-1 / n} \leq-\frac{d}{d t} \int_{|u|>t}|D u| d x .
$$

Use Hölder's inequality on the right-hand side of (3.2) and recall

(3.1) to get

$$
\begin{aligned}
& \left(n C_{n} \mu(t)^{1-1 / n}\right)^{2} \\
& \leq\left(-\frac{d}{d t} \int_{|u|>t} \frac{f^{2}(x)}{m(x)} d x\right)\left(-\frac{d}{d t} \int_{|u|>t} \frac{1}{m(x)} d x\right) .
\end{aligned}
$$


According to Lemma 2.1 consider a function $F^{2}(s)$ and a function $1 / \underline{m}(s)$ such that

$$
\int_{|u|>t} \frac{f^{2}(x)}{m(x)} d x=\int_{0}^{\mu(t)} F^{2}(s) d s
$$

and

$$
\int_{|u|>t} \frac{1}{m(x)} d x=\int_{0}^{\mu(t)} \frac{1}{\underline{m}(s)} d s .
$$

Then use (3.3) to see that

$$
1 \leq \frac{1}{n C_{n}^{1 / n}} \frac{F(\mu(t))}{\underline{m}^{1 / 2}(\mu(t))} \mu(t)^{1 / n-1}(-\mu(t)) .
$$

Integrate the above between 0 and $t$ and recall the definition of $u^{*}(s)$ to obtain

$$
u^{*}\left(c_{n}|x|^{n}\right) \leq \frac{1}{n c_{n}^{1 / n}} \int_{C_{n}|x|^{n}}^{|\Omega|} \frac{F(r)}{\underline{m}^{1 / 2}(r)} r^{-1+1 / n} d r=v(x) .
$$

THEOREM 3.1. Let (i), (ii) be satisfied, $q>n$ and $p=n(q-1) /(q-n)$, then

$$
[u]_{1-1 / n} \leq\left(n c_{n}\right)^{-1 / n}\left\|m^{-1}\right\|_{q}^{1-1 / p}\left\|f m^{-1 / p}\right\|_{p} .
$$

Proof. By definition of $[u]_{1-1 / n}$ (see $(1.7)$ ) and by Lemma 3.1 we have

$$
[u]_{1-1 / n} \leq[v]_{1-1 / n}
$$

On the other hand

$$
\begin{aligned}
& {[v]_{1-1 / n}^{n} \leq\left(n C_{n}\right)^{-1}\|D v\|_{n}^{n} \quad \text { by }(1.8)} \\
& =\left(n C_{n}\right)^{-1}\left\|F\left(C_{n}|x|^{n}\right) / \underline{m}^{1 / 2}\left(C_{n}|x|^{n}\right)\right\|_{n}^{n} \text { by definition of } v(x) \\
& =\left(n C_{n}\right)^{-1} \int_{0}^{|\Omega|}\left|\frac{F^{2}(s)}{\underline{m}(s)}\right|^{n / 2} d s .
\end{aligned}
$$

Now because of Lemma 2.1 there exists a sequence $\left\{\psi_{k}^{2}(s)\right\}$ of functions equidistributed with $f^{2} / m$ and such that

$$
\psi_{k}^{2}(s) \longrightarrow F^{2}(s)
$$


in $L^{\alpha}(0,|\Omega|)$, with

$$
\frac{1}{\alpha}=\frac{2}{p}+\frac{1}{q} \frac{p-2}{p}
$$

Hence

$$
\begin{aligned}
& \int_{0}^{|\Omega|}\left|F^{2}(s)\right|^{\alpha} d s \leq \underset{k}{\lim } \int_{0}^{|\Omega|}\left|\psi_{k}^{2}(s)\right|^{\alpha} d s \\
& =\int_{0}^{|\Omega|}\left(\left(\psi_{k}^{2}(s)\right)^{*}\right)^{\alpha} d s=\int_{\Omega}\left(\frac{f^{2}(x)}{m(x)}\right)^{\alpha} d x,
\end{aligned}
$$

by using the well known result (see [5], [10], [18])

$$
\int_{\Omega} u(x) d x=\int_{0}^{|\Omega|} u *(s) d s \text {. }
$$

Analogously by Lemma 2.1 there exists a sequence $\left\{\zeta_{k}(s)\right\}$ equidistributed with $m^{-1}(x)$ and such that

in $L^{q}(0,|\Omega|)$. Thus

$$
\zeta_{k}(s) \rightarrow \frac{1}{\underline{m}(s)}
$$

$$
\int_{0}^{|\Omega|} \frac{1}{\underline{m}^{q}(s)} d s \leq \lim _{k} \int_{0}^{|\Omega|}\left|\zeta_{k}(s)\right|^{q} d s=\int_{\Omega} \frac{1}{m^{q}(x)} d x .
$$

Now, $\frac{n}{2 \alpha}+\frac{n}{2 q}=1$ since $p=n(q-1) /(q-n)$, and hence by Hölder's inequality and by (3.6) and (3.7)

$$
\begin{aligned}
& \int_{0}^{|\Omega|}\left(\frac{E^{2}(s)}{\underline{m}(s)}\right)^{n / 2} d s \\
& \leq\left\{\int_{\Omega}\left(\frac{f^{2}(x)}{m(x)}\right)^{\alpha} d x\right\}^{n / 2 \alpha}\left\{\int_{\Omega} \frac{1}{m^{q}(x)} d x\right\}^{n / 2 q} \\
& \leq\left\|f m^{-1 / p}\right\|_{p}^{n} \cdot\left\|m^{-1}\right\|_{q}^{n(p-1) / p}
\end{aligned}
$$

(again by Holder's inequality since $\frac{2 \alpha}{p}+\frac{\alpha}{q} \frac{p-2}{p}=1$ ).

Now the above inequality with $(3.4)$ and (3.5) gives the required estimate. 
Remarks. (1) At least for the uniformly elliptic case $(m=1)$, the constant in Theorem 3.1 is the best possible.

(2) An obvious consequence of Theorem 3.1 and (1.9) is that

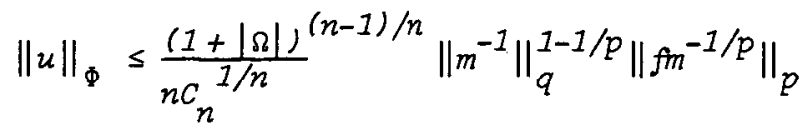

with $\Phi(t)=\exp \left(|t|^{n /(n-1)}\right)-1$.

(3) Theorem 3.1 improves the estimate (b) of Theorem 7.2 in [14] and th estimate obtained in Theorem 4.1 of [21] for case II.

(4) Using the Comparison Lemma 3.1 we can derive the numerical value of the constants in "sharp" estimates for a solution $u$ of (1.1) by known methods ([4], [18], [8]).

For example we have for $m=1$ and $p>n$ :

$$
\|u\|_{\infty} \leq K_{1}(n, p)|\Omega|^{\frac{1}{n}-\frac{1}{p}}\|f\|_{p}
$$

where

$$
K_{1}(n, p)=\frac{1}{n C_{n}^{1 / n}}\left[\frac{n(p-1)}{p-n}\right]^{1-1 / p}
$$

This constant is the best one as already known (see [22]).

For $m=1$ and $2 \leq p \leq n$, via the Bliss inequality (see [18])

$$
\int_{0}^{\infty}\left(\int_{r}^{\infty} \psi(s) d s\right)^{q} d r \leq B\left(\int_{0}^{\infty} \psi(r)^{p_{r}-1+p+p / q} d r\right)^{q / p} q>p>1
$$

where

$$
B=\frac{B}{q(p-1)}\left\{\frac{\Gamma(p q /(q-p))}{\Gamma(q /(q-p)) \Gamma(p(q-1) /(q-p)}\right\}^{(q / p)-1}(q(1-1 / p))^{q-(q / p)+1}
$$

we get:

$$
\|u\|_{p^{*}} \leq K_{2}(n, p)\|f\|_{p}
$$

where

$$
K_{2}(n, p)=\frac{1}{n c_{n}^{1 / n}}\left\{\frac{\Gamma(n)}{\Gamma(n / p) \Gamma(1+n-n / p)}\right\}^{1 / n}\left\{\frac{n(p-1)}{n-p}\right\}^{1-1 / p}
$$

Also this constant is the best possible one ([19]) . 


\section{References}

[1] R.A. Adams, Sobolev Spaces, (Academic Press, New York, San Francisco, London, 1975).

[2] A. Alvino, "Un caso limite della diseguaglianza di Sobolev in spazi di Lorentz", Rend. Accad. Sci. Fis. Mat. Napoli XLIV (1977), 105-112.

[3] A. Alvino, P.L. Lions and G. Trombetti, "On optimization problems with prescribed rearrangements", (to appear).

[4] A. Alvino and G. Trombetti, "Sulle migliori costanti di maggiorazione per una classe di equazioni ellittiche degeneri", Ricerche Mat., XXVII (1978), 413-428.

[5] C. Bandle, Isoperimetric Inequalities and Applications, (Pitman, London 1980).

[6] E. De Giorgi, "Su una teoria generale della misura ( $r-1)$ dimensionale in uno spazio ad $r$ dimensioni", Ann. Mat. Pura Appl., 36 (1954), 197-213.

[7] W. Fleming and R. Rishel, "An integral formula for total gradient variation", Arch Math., 11 (1960).

[8] E. Giarrusso and D. Nunziante, "Symmetrization in a class of first-order Hamilton-Jacobi equations", Nonlinear Anal. 8 (1984), 289-299.

[9] D. Gilbarg and N.S. Trudinger, Elliptic Partial Differential Equations of Second Order, (Springer-Verlag, Berlin, Heidelberg, New York, Takyo, 1983).

[10] B. Kawoh1, Rearrongements and Convexity of Level Sets in P.D.E., (Lecture Notes in Mathematics, 1150, Springer-Verlag, Berlin, Heidelberg, New York, Tokyo, 1985).

[11] M.A. Krasnosel'skii and Y.A. Rutickii, Convex Functions and Orlicz Spaces, (P. Noordhoff Ltd., Groningen, 1961).

[12] L. Migliaccio, "Sur une condition de Hardy-Littlewood-Polya", C.R. Acad. Sci. Paris, Sér. I. Math. 297 (1983), 25-28.

[13] J. Moser, "A sharp form of an inequality by N. Trudinger", Indiana Univ. Math. J., 20 (1971), 1077-1092.

[14] M.K.V. Murthy and G. Stampacchia, "Boundary value problems for some degenerate elliptic operators", Ann. Math. Pura Appl., 90 (1971), 1-122. 
[15] J. Ryff, "Majorized functions and measure", Indag. Math. 30 (1968), 431-437.

[16] G. Stampacchia, "Le problème de Dirichlet pour les êquations elliptiques du second ordre à coefficients discontinus", Ann. Inst. Fourier (Grenoble) 15 (1965), 189-258.

[17] G. Stampacchia, "Some limit cases of $L^{p}$-estimates for solutions of second order elliptic equations", Comm. Pure App Z. Math. XVI (1963), 505-510.

[18] G. Talenti, "Elliptic equations and rearrangements", Ann. Scuola Norm. Sup. Pisa Cl. Sci. 3 (1976), 697-718.

[19] G. Talenti, "Best constant in Sobolev inequality", Ann. Mat. Pura App 2. 110 (1976), 353-372.

[20] N.S. Trudinger, "On imbeddings into orlicz spaces and some applications", J. Math. Mech., 17 (1967), 473-484.

[21] N.S. Txudinger, "Linear elliptic operators with measurable coefficients", Ann. Scuola Norm. Sup. Pisa Sci. Fis. Mat., 27 (1973), 265-308.

[22] H.F. Weinberger, "Symetrization in uniformly elliptic problems", Studies in Math. Analysis and related topics, (stanford Univ. Press, Stanford, California, 1962), 424-428.

Dipartimento di Matematica ed Applicazioni

"R. Caccioppoli", Universita di Napoli

Via Mezzocannone, 8; 80134 Napoli

Italia 